\title{
HIGH-SENSITIVITY MASS SPECTROMETRY WITH A. TANDEM ACCELERATOR
}

\author{
Walter Henning \\ Argonne National Laboratory, Argonne, 111 inois 60439 \\ and \\ Physik-Department der Technischen Universität München \\ 8046 Garching. West-Germany
}

\section{DISCLAIMER}

This report was prepared as an account of work sponsored by an agency of the United States Government. Neitier the United States Government nor any agency thereof, nor any of their empioyees, makes any warranty, express or implied, or assumes any legal liability or responsibility for the accuracy, completeness, or usefulness of any information, apparatus, product, or process disclosed, or represents that its use would not infringe privately owned rights. Reference herein to any specific commercial product, process, or service by trade name, trademark, manufacturer, or otherwise does not necessarily constitute or imply its endorsement, recommendation, or favoring by the United States Government or any agency thereof. The views and opinions of authors expressed herein do not necessarily state or reflect those of the United States Government or any agency thereof.

CONF $-830384--2$

DE33 017914

\section{NOTICE}

PORTIONS IJF TUSS REPORT ARE ILLEGBLE.'

It has been reproduced from the best available copy to permit the broadest possible availability. 
HIGH-SENSITIVITY MASS SPECTROMETRY WITH A TANDEM ACCELERATOR

Walter Henning

Argonne National Laboratory. Argonne, Illinois 60439

and

Physik-Department der Technischen Universität München

8046 Garching, West-Germany

Abstract

The characteristic features of accelerator mass spectrometry are discussed. A short overview is given of the current status of mass spectrometry with high-energy (Mev/nucleon) heavy-ion accelerators. Emphasis is placed on studies with tandem accelerators and on future mass spectrometry of heavier isotopes with the new generation of higher-voltage tandems.

\section{NOTGE}

PORTIONS OF TEIS REDTRT ORE IERERIBLE:

It has heen leprodilced frem the best

\section{Introduction} available copy to permit the broadest possible availability.

Accelerators have provided beams for most of the measuremerits presented at this symposium. While essential to a sucessiul experiment, they are not necessarily considered an inherent part of the experimental set-up. Quite the opposite is true in experimeits of accelerator mass spectrometry (AMS): Here the accelerator becomes the central part of the experimental configuration, aided by an ion-sourca at the injection end, and a heavy-ion spectrometer after the accelerator. Configurations like the one schematically shown in Figure 1, have over the past six years ${ }^{1)}$ developed to mass spectrometers with sensitivities (up to $1: 10^{15}$ ) that can considerably exceed those of conventional, low-energy mass spectroneters $\left(\sim 1: 10^{8}\right)$. The basic reason is the capability in high-energy spectrometry to individually iden- 
tify accelerated ions by mass $M$ and nuclear charge $Z$. One easily calculates that if a single ion $c a n$ be identified and

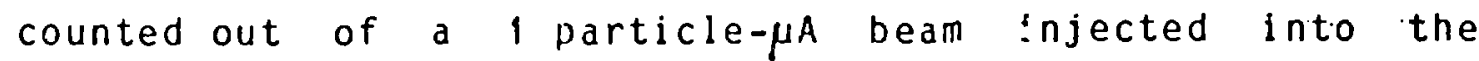
accelerator and transmitted with $10 \%$ probability, over a period of one hour a sensitivity of $\sim 1: 10^{15}$ is achieved.

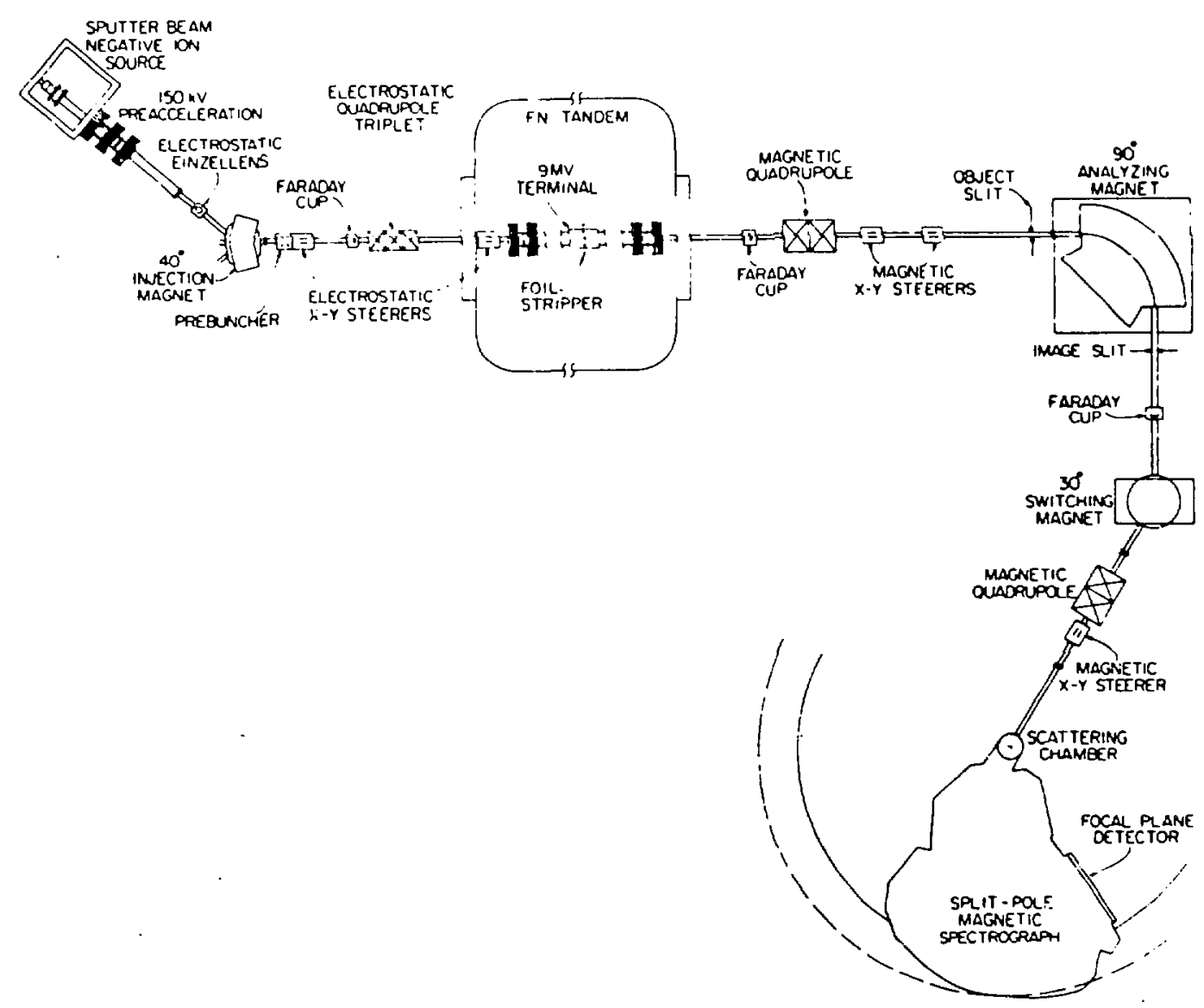

Fig. 1: The Argonne FN Tandem accelerator system as an example for a tandem facility used in accelerator mass spectrometry (AMS). A negative-ion beam from a sputter beam ion source (at left) is injected into the tandem, stripped at the terminal, and the radioisotopes of interest are selected and identified in an all-magnetic beam-line system and spectrograph at the high-energy end of the accelerator. (Figure from reference 9 , where additional technical details can be found). 


\section{Characteristics of AMS}

A number of properties of ion source, accelerator and heavy-ion spectrometer contribute to the success of AMS. Some are specific to particular isotopes, as the lack of negative-ion formation for interfering isobars (cr their molecules) as for example for $14_{\mathrm{N}}\left({ }^{14} \mathrm{C}\right),{ }^{26} \mathrm{Mg}\left({ }^{26} \mathrm{Al}\right)$, ${ }^{36} \mathrm{Ar}\left({ }^{36} \mathrm{Cl}\right)$ or $\left.{ }^{4}{ }^{1} \mathrm{KH}_{3}\left({ }^{4} \mathrm{CaH}_{3}\right)^{2}\right)$. But the more general advantages of high-energy ( $\sim$ MeV/nucleon) mass spectrometry over conventional dow-energy ( $\sim$ keV/nucleon) mass spectrometry

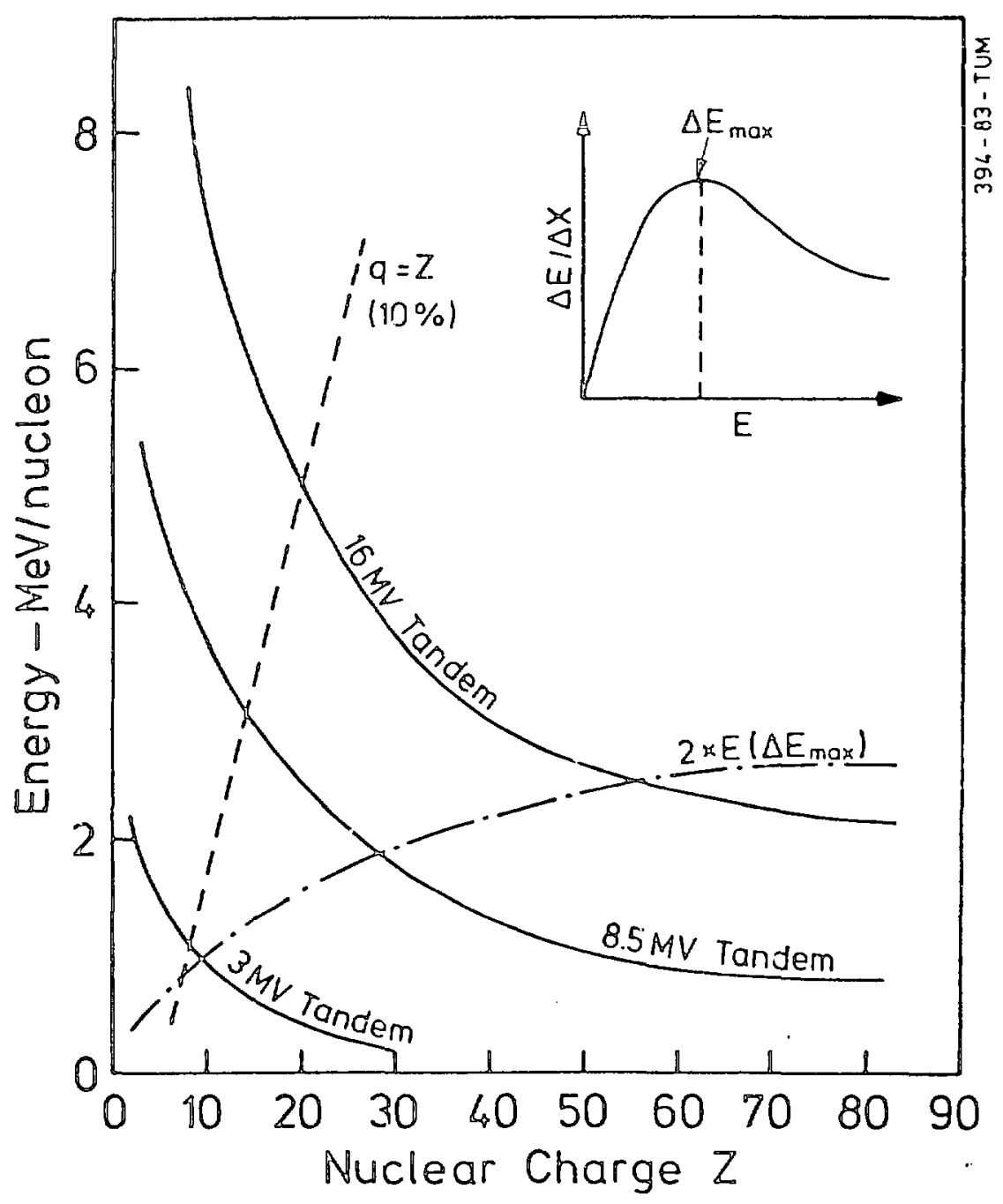

Fig. 2: Energy curves relevant for high-energy AMS (see text). 
arlse from a highe: kinetic energy: i) through break-up of isobaric molecules in thin foils which have otherwise no adverse effect on the high-energy radioisotope ion af interest. il) from icientification and separation of isobars through nuclear charge determination $v i a d E / d x$ measurements, and $i \dot{i}$ i) from reduced slit scattering cross section $\left(\alpha E^{-2}\right)$. of these three。 $i)$ and $i j i)$ are less dependent on the exact kinetic energy avallable, but ij) requires a certain minimum energy determined in principal by the fact that the differential energy-loss curves overlap at lower energies, and in practice by the requirement that for reasonable separation of neighbouring elements one needs incident energies two or three times the value at which $d E / d x$ has its maximum.

This has consequences for the range of nuclei that can be investigated through AMS with a specific accelerator. Figure 2 qualitatively describes the situation for relevant energies as a function of nuclear charge $z$ : The solid curves labeied $3 \mathrm{MV}, 8.5 \mathrm{MY}$, and $16 \mathrm{MV}$ indicate the maximum energles (with reasonable intensities) achievable with tandems of corresponding terminal voltage. Also shown is a curve indicating twice the energy where $d E / d x$ has a maximum; the curve for all practical purposes indicates the minimum energy necessary for separation of neighbouring $Z^{\prime} s$. One recognizes that the projected dedicated facilities ${ }^{1}{ }^{2}$ for AMS ( 3 MV) are limited to rather $\mathbb{l}$ ight isotopes, the oftenused $F N$ tanciems ( $\sim 8.5 \mathrm{MV}$ ) allow measurements maybe up to ${ }^{4} \mathrm{Ca}$. whereas a 16 MV tandem as the one becoming operational at the host institution of this symposium, Legnaro, can nearly double the range of nuclear charge accessible and extend into a new regime of medium-weight radioisotopes, as discussed in more detail below. The energy curves shown in figure 2 are for a 1 practical purposes minimum energies. In particular when absorber foils are used to outrange and eliminate interfering isobars of lower $z$ considerably higher energies are necessary (see for example measurements of ${ }^{10} \mathrm{Be}$ in reference 11 . Also shown in Figure 2 is a curve for the 
approximate energy necessary to fully strip a radioisotope with $10 \%$ probability. The technique of fully stripping a radioisotope has been successfully used to eliminate interfering stable isobars with lower nuclear charge ${ }^{3}$.)

\section{Some Technical Aspects}

Various types of heavyoion accelerators have been used in AMS-type studies, as indicated in the (most likely incomplete) table below. Most of the actual applications, however. have been performed with two accelerator types: the cyclotron and the tandem: and it probably fair to say that the bulk of these studies was done at tandems. Work at other accelerators constitutes feasibility studies of these machines for AMS, or seiected measurements as for example the search for fractionally charged particles ${ }^{4)}$.

Table: Heayy-Ion Accelerators used in AMS-type Studies.

Cyclotron:

Tandem

EN:

FN:

MP:

SQUD:

3MV (ded):

Linac:

Cockcroft-Walton:

Dynamitron:
Berkeley, Grenoble, Orsay

\section{Zurich}

Argonne, Mcllaster. Pennsylyania,

Rutgers, Saclay, Seattle

Chalk River, Munich, Orsay.

Rochester, Yale

Rehovot

Arizona, Nagoya, Oxford, Toronto, Gifsur-yvette

Argonne. Munich, Orsay

Fermilab

Argonne 
The domiance of tandems results from their widespread use in nuclear physics but also from the fact, that their technical layout - designed long before they were considered for AMS studies - has features very favorable for AMS. This is illustrated in Figure 1 with the example of the Argonne FN tandem. which has been used extensively in AMS measurements with essentially no tecnnical components added. The low-energy end is all-electrostatic, thus independent of the mass of the singly-charged negative ions, except for the injection magnet which selects ine isotope mass of interest. The high-energy end is essentially all-magnetic, with identical ion optics for ions of same magnetic rigidity. Therefore the measurement of a radioisotope concentration in a source sample. which is done by comparing the radioisotope rate at the heavy-ion spectrometer with that of a stable isotope from the same source sample (generally a macroscopic ion-beam measured in a Faraday cup at the location of the heayy-ion spectrometer), requires only two changes: that of the injection magriet to switch between radioisotope and stable-isotope mass and that of the terminal voltage to produce constant magnetic rigidity for both ion species at the high-energy end.

\section{Applications of AMS}

Measurements of yery low concentraijons of radioisotopes in AMS have allowed studies in various fields that were difficult or sometimes impossible to perform otherwise. These include measurements of nuclear lifetimes and nuclear cross sections, search for exotic particles and a large number of dating and tracing applications in geology, archeology, hydrology, cosmology to name just a few. It is beyond the scope of this paper to give a complete overview, the reader is referred to the proceedings of three recent conferences 1 ). The extend of these studies can be seen. for example. from a list of AMS measurements of cosmogenic ${ }^{10} \mathrm{Be}$ 
compiled in a recent article by $W$. Kutschera 5) and reproduced in Figure 3 .

AMS MEASUREMENTS OF COSMOGENIC BO

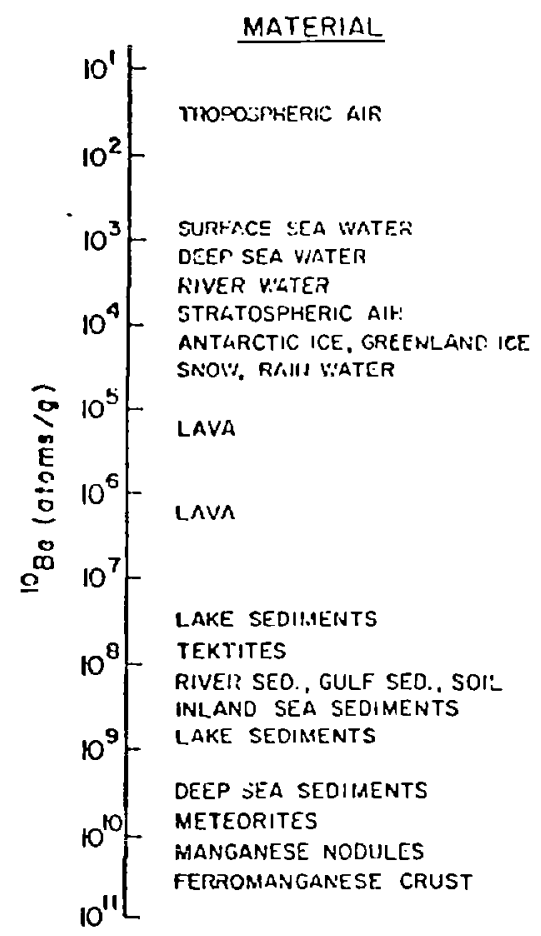

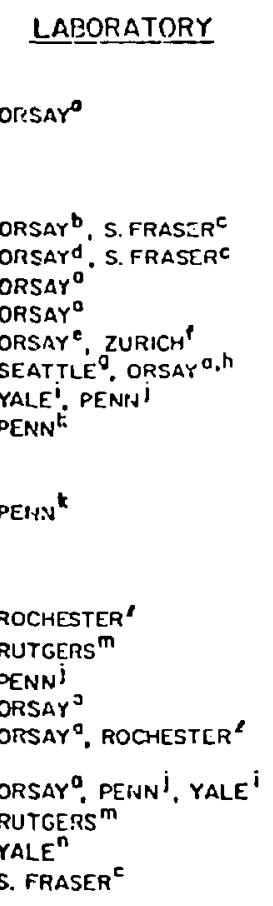

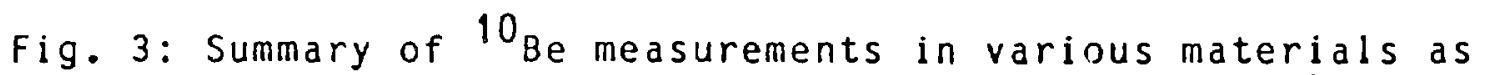
compiled in a recent article by $W$. Kutschera ${ }^{5}$.

V. Future Extension of AMS to Medium-Mass Radioisotopes

Since this symposium is being held at an institution where a tandem of higher terminal-voltage is becoming operational, it seems appropriate to discuss future extensions of AMS to heavier radioisotopes. There is great interest in several of these as for example in ${ }^{41} \mathrm{Ca}, \mathrm{a}$ constituent of fossile bones, with a halflife of $10^{5}$ years. ${ }^{41} \mathrm{Ca}$ might allow to extend dating of fossiles into the time range inaccessible with ${ }^{14} \mathrm{C}$ dating. The natural concentration is expected to be very low $\left(\sim 10^{-15}\right)$ and considerable scepticism is in order whether it will be possible to utilize ${ }^{41} \mathrm{Ca}$ in AMS studies. We have had some encouraging 
results at the MP tandem in Munich recently ${ }^{6)}$, where the combined use of an existing Wien-Filter after the MP and a Q30 heavy-ion spectrograph has allowed us to detect ${ }^{41} \mathrm{Ca}$ radioisotope ions from enriched samples, prepared through neutron activation of ${ }^{40} \mathrm{Ca}$ in a reactor, with good efficiency and, at present, with a lower sensitivity of a few times $10^{-14}$. For natural samples we believe that a preenrichment of a factor 100, feasible with a conventional mass separator, will allow detailed AMS studies with ${ }^{41} \mathrm{Ca}$.
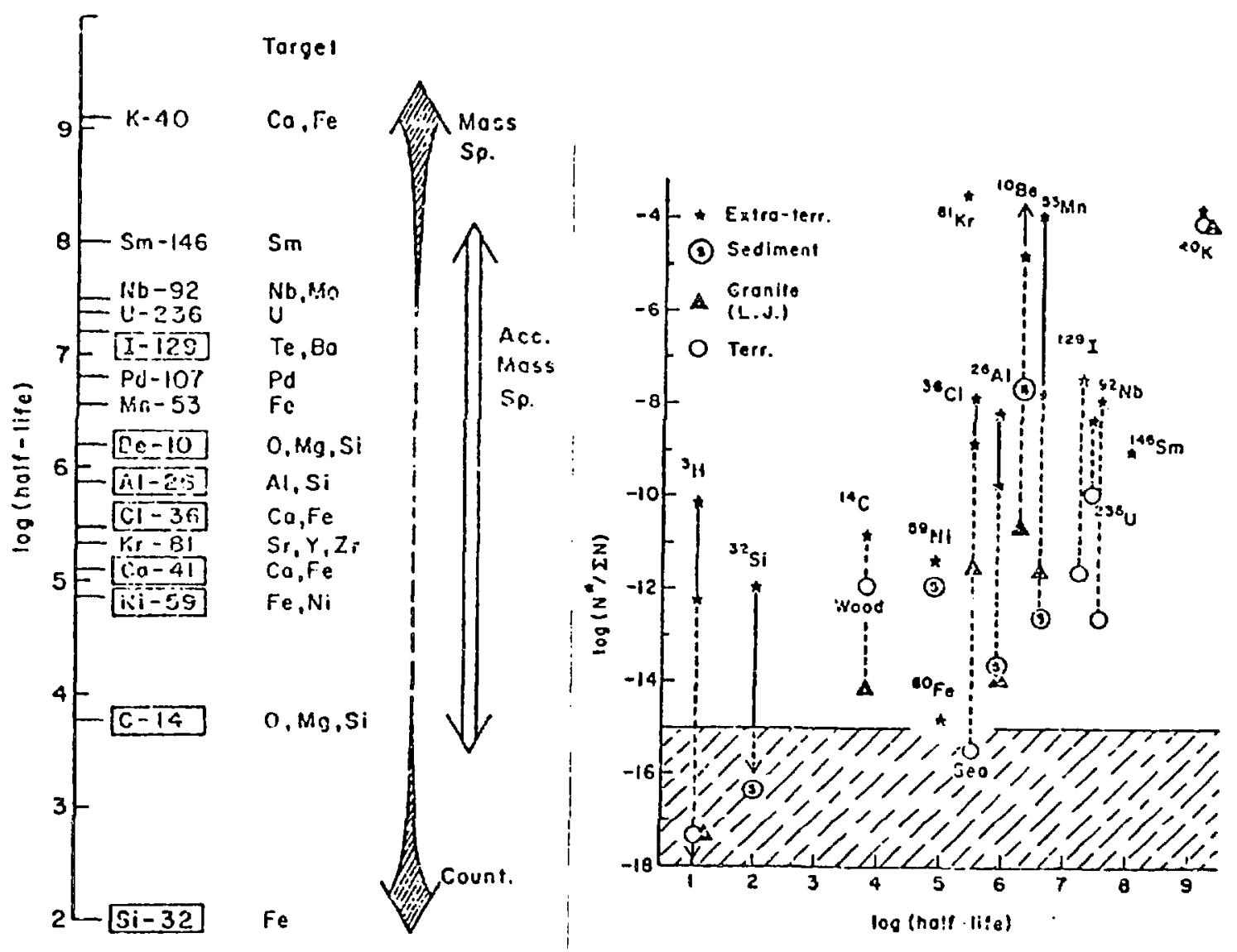

Fig. 4: Left: Cosmogenic nuclides with various half-lives. Nuclides enclosed in boxes have been studied by AMS. Right: Ratio of radioisotope to stable-isotope abundance $\left(N^{*} / N\right)$ in various samples. The hatched area shows typical detection limits at present. (From ref. 7). 
other heavy radioisotopes of cosmogenic origin are of considerable interest (e.g. ${ }^{53} \mathrm{Mn},{ }^{59} \mathrm{Ni},{ }^{60} \mathrm{Fe},{ }^{92} \mathrm{Nb}$, etc) as pointed out in a recent article by Nishizumi and. Arnold?) from which Figure 4 is taken. Not all of them require AMS techniques (for example ${ }^{53} \mathrm{Mn}$ was detected at low concentrations by the authors of reference 7 through neutron activation studies).

A successful measurement of a heary radioisotope, ${ }^{44} \mathrm{Ti}$, with AMS has been completed recently at Argonne 81 . Figure 5 shows isometric spectra of energy loss $\triangle E$ versus total energy $E$ as measured for two $T i$ ion-source samples, one spiked with ${ }^{44} \mathrm{Ti}$ at a concentration of $2 \times 10^{-7}$. The
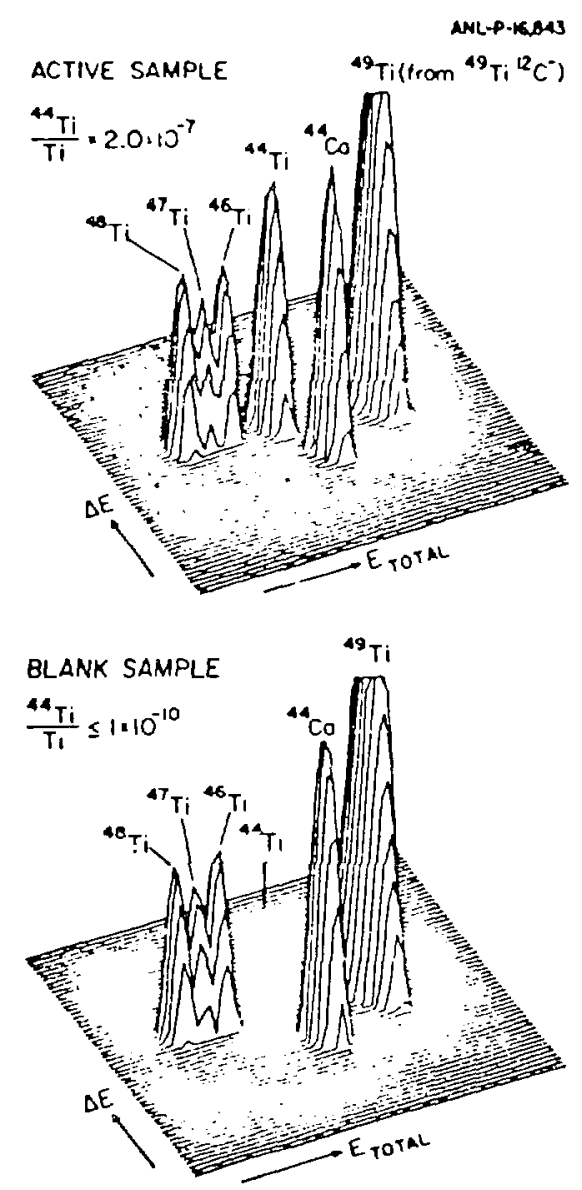

Fig. 5: Energy loss versus total energy of ions detected by the focal plane detector of the Argonne splitpole magnetic spectrograph for ${ }^{44} \mathrm{Ti}$ ion "beams" from an active and a blank $\mathrm{Ti}$ source sample. 
results together with activity measurements, allowed a quite accurate determination of the ${ }^{44} \mathrm{Ti}$ half-1 ife: $T_{1 / 2}=54.2 \pm$ 2.1 jears.In these measurements absolute ${ }^{44} \mathrm{Ti} / \mathrm{Ti}$ ratios had to be determined (in contrast to ratios determinec from a comparison with a calibrated reference sample), and the relatively small error is a result of careful measurements of mass fractionation effects usirj the various stable isotopes of $T i$. The measurement, though only performed at the low energies available from the Argonne $F N$ tandem, revealed a limiting sensitivity of $10^{-10}$ for this heavy radioisotope. clearly not sufficient for the limits necessary to sensibly use AMS in this mass range for natural (terrestrial or cosmogenic) concentrations. But encouraging in the sense that AMS works well in principle for the heavier masses and should provide sufficiert sensitivity when Improved backgrould reduction is achieved with higher-energy beams from tandems with sufficiently high terminal voltages. There is clearly a large number of interesting problems tc be solved where a high-energy tandem. as the one becoming operational here at Legnaro, can make unique contributions.

This work was supported in part by the U.S. Dept. of Energy under contract number $W-31-103-E N G-33$ and in part by the Bundesministerium für Forschung und Technologie, Bonn. 
4. Proc. ist Confo Radiocürbon Dating with Accelerators, editor HoT. Gove, Rochester (1978): Proc. Symp. on Accelerator Mass Spectrometry, editors $W$. Henning, $H_{0}$ Kutschera, R.K. Smither, and J.L. Yntema. Argonne National Laboratory Report ANL/ PHY-81-1 (9981): Proc. Pitio Int. Radiocarbon Conf.., Seattle (1982), editors M. Stuiver and R.S. Kra, to be published in Radiocarbon, Vol. 25.

2. GoM.Raisbeck.F. Yiou,A. Peghaire, J. Gulllot, and J. Uzureau, Proc. Symp. on Accelerator Mass Specircmetry. Argonne National Laboratory Report ANL/PHY-81-8 (1981)。? 426。

3. G.M. Raisbeck and Fo Yiou, J。 de Physique 40, L-241, and to be published in Revue $\mathrm{H}$ : Arche'metrie: $W_{0}$ Henning, $W_{0}$ Kutschera, B. Myslek-Laurikainevi, R.C. Pardo, RoK. Smither, and J.L. Yntema, Proc. Symp. on Accelerator Mass Spectrometry, Argonne Nationa! Labo-atory Report ANL/PHY-81-1 (8981).P. 320; S. Gustavsson, Go Korschinek, P.W. Klibik, H. Morinaga, E。 Noite, and MoS. Pravikoff, Annual Report Tandem Laboratory. Munich (1981), see also Vernandlungen JPG $5=4036$ (1983).

4. J.P. Schiffer, D. Frekers, $W_{0}$ lienning, W. Kut schera, K.H. Shepard, C. Curtis, and C. Schmidt, Buil. Am。 Phys,Soc。 28 (1983) 709. 
5. W. Kutschera, Proc. 11 th Int. Radiocarbon Conf., Seattle (1982). editors M. Stuiver and R.S. Kra,to be published in Radiocarbon, Vol. 25.

ט. P. Hille, W. Henning, H. Ernst, G. Korschinek, P.W. Kubik, W. Mayer, E. Nolte, and H.J. Scheerer, Munich (1983), to be published.

7. K. Nishizumi and J.R. Arnold, Proc. Symp. on Acceieracor Mass Spectrometry, Argonne National Laboratory Report AilL/PHY-81-1, p. 262.

8. D. Frekers, W. Henning, W. Kutschera, K.E. Rehm, R.K. Smither, J.L. Yntema, R. Santo, B. Stievano, and $N$. Trautmann, to be published; see also Bull. Am. Phys. Soc. 28 (1982) 714.

9. W. Herning, W. Kutschera, M. Paul, R.K. Smither, E.J. Siephenson, and J.L. Yntema, Nucl. Instr. and Metr. 184 (ig8!) 247. 